\title{
A High-Granularity Timing Detector for the Phase-II upgrade of the ATLAS Calorimeter system: detector concept, description and R\&D and beam test results
}

\author{
H. Imam on behalf of the ATLAS HGTD Collaboration * \\ HASSAN II University - Faculty of Sciences Ain Chock \\ Casablana,Morocco \\ Corresponding author: hajarimam@cern.ch
}

\begin{abstract}
The particle flux increase (pile-up) at the HL-LHC with luminosities of $\mathbf{L}=7.5 \times 10^{34} \mathrm{~cm}^{-2} \mathrm{~s}^{-1}$ will have a significant impact on the reconstruction of the ATLAS detector and on the performance of the trigger. The forward region and the end-cap where the internal tracker has poorer longitudinal track impact parameter resolution, and where the liquid argon calorimeter has coarser granularity, will be significantly affected. A High Granularity Time Detector (HGTD) is proposed to be installed in front of the LAr end-cap calorimeter for the mitigation of the pileup effect, as well as measurement of luminosity.

It will have coverage of 2.4 to 4.0 from the pseudo-rapidity range. Two dual-sided silicon sensor layers will provide accurate timing information for minimum-ionizing particles with a resolution better than $30 \mathrm{ps}$ per track (before irradiation), for assigning each particle to the correct vertex. The readout cells are about $1.3 \mathrm{~mm} \times 1.3 \mathrm{~mm}$ in size, which leads to a high granular detector with 3 million channels. The technology of low-gain avalanche detectors (LGAD) with sufficient gain was chosen to achieve the required high signal-to-noise ratio. A dedicated ASIC is under development with some prototypes already submitted and evaluated. The requirements and general specifications of the HGTD will be maintained and discussed. R\&D campaigns on the LGAD are carried out to study the sensors, the related ASICs and the radiation hardness. Both laboratory and test beam results will be presented.
\end{abstract}

Index Terms-HL-LHC, ATLAS, HGTD, LGAD, Si sensors, Timing detectors

\section{INTRODUCTION}

The HL-LHC [1] is an upgrade of the LHC project, which will deliver an integrated luminosity of up to $4000 \mathrm{fb}^{-1}$ over the subsequent decade, and achieve an instantaneous luminosity of $\mathrm{L}=7.5 \times 10^{34} \mathrm{~cm}^{-2} \mathrm{~s}^{-1}$, compared to the current value of $\mathrm{L}=2.5 \times 10^{34} \mathrm{~cm}^{-2} \mathrm{~s}^{-1}$. This will increase the pileup interactions to an average of 200 simultaneous proton-proton interaction within the same bunch crossing interval $(\langle\mu\rangle=$ 200), which is 6 times higher than the current pileup rate. The Inner Tracker upgrade (ITk) [2] will have a better position resolution and pseudo-rapidity extended to $|\eta|=4.0(\eta<$

* Copyright 2021 CERN for the benefit of the ATLAS Collaboration. Reproduction of this article or parts of it is allowed as specified in the CCBY-4.0 license.
2.5 up to now), but the ITk will have low $\mathrm{Z}_{0}$ resolution in the forward region. So the timing information can help to further reduce pileup effect in this region. Hence by using only the ITk, multiple tracks nearby in the space can lead to track/vertex association ambiguities. The timing information of the HGTD [3] reduces the density of vertices for a given track, and allows the effective separation of the hard scatter vertex from pile-up vertices surrounding it in the $\mathrm{Z}$ direction, hence the improvement of the ATLAS [4] performance in the forward region.

\section{HGTD REQUIREMENTS AND LAYOUT}

\section{A. Overview \& requirements}

To ensure the performances needed to deal with the high luminosity expected in the HL-LHC phase $(\langle\mu\rangle=200)$, a part of the ATLAS [4] detector has to be upgraded, where the electromagnetic calorimeter [5] has larger granularity and the inner tracker resolution degrades. Consequently, The HGTD detector which will provide a high granularity and timing information is proposed to be located in the gap region between the end-cap calorimeters and the barrel. Each HGTD end-cap is the integration of one hermetic vessel, two instrumented double sided layers and two moderators placed inside also outside the airtight vessel. The modules with sensors and on-detector electronics are mounted on the front and back sides of a common cooling disk. Each disk of this last is physically separated in two half circular disks. The HGTD will cover the pseudo-rapidity range between $2.4<|\eta|<4$.0, complementing the inner tracker by providing high-precision time measurements for charged particles, and enhancing the performance of physics object reconstruction. Fig. 1 shows the position of the HGTD detector.

Due to space limitations and the high expected radiation level at the HGTD position, a technology of silicon-based time sensor is selected to be used, with thin and configurable array sensors. These requirements are satisfied using the Low Gain Avalanche Detector (LGAD) [6] pads of $1.3 \mathrm{~mm} \times 1.3 \mathrm{~mm} \times$ $50 \mu \mathrm{m}$ (length $\times$ width $\times$ thickness). Such pad dimensions ensure under $10 \%$ of occupancy at high pileup ranges, limited 


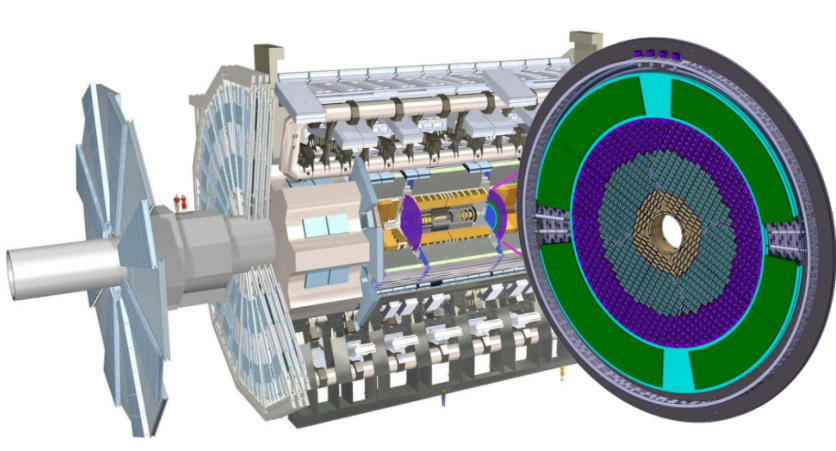

Fig. 1: Position of the HGTD within the ATLAS Detector [3].

\begin{tabular}{|c|c|}
\hline $\begin{array}{l}\text { Pseudo-rapidity coverage } \\
\text { Thickness in } \mathrm{z} \\
\text { Position of active layers in } \mathrm{z} \\
\text { Weight per end-cap } \\
\text { Radial extension: } \\
\quad \text { Total } \\
\quad \text { Active area }\end{array}$ & 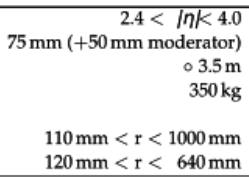 \\
\hline $\begin{array}{l}\text { Pad size } \\
\text { Active sensor thickness } \\
\text { Number of channels } \\
\text { Active area } \\
\text { Module size } \\
\text { Modules }\end{array}$ & $\begin{array}{r}1.3 \mathrm{~mm} \times 1.3 \mathrm{~mm} \\
50 \mu \mathrm{m} \\
3.6 \mathrm{M} \\
6.4 \mathrm{~m}^{2} \\
30 \times 15 \text { pads }(4 \mathrm{~cm} \times 2 \mathrm{~cm}) \\
8032\end{array}$ \\
\hline Collected charge per hit & $>4.0 \mathrm{fC}$ \\
\hline $\begin{aligned} & \text { Average number of hits per track } \\
& 2.4<\operatorname{lnK} 2.7(640 \mathrm{~mm}>\mathrm{r}>470 \mathrm{~mm}) \\
& 2.7<\operatorname{lnK} 3.5(470 \mathrm{~mm}>\mathrm{r}>230 \mathrm{~mm}) \\
& 3.5<\operatorname{lnK} 4.0(230 \mathrm{~mm}>\mathrm{r}>120 \mathrm{~mm})\end{aligned}$ & $\begin{array}{l}\approx 2.0 \\
\approx 2.4 \\
\approx 2.6\end{array}$ \\
\hline $\begin{array}{l}\text { Average time resolution per hit (start and end of operational lifetime) } \\
\quad 2.4<I \eta K 4.0 \\
\text { Average time resolution per track (start and end of operational lifetime) }\end{array}$ & $\begin{array}{l}\approx 35 \mathrm{ps}(\text { start }), \approx 70 \mathrm{ps} \text { (end) } \\
\approx 30 \mathrm{ps}(\text { start }), \approx 50 \mathrm{ps}(\text { end })\end{array}$ \\
\hline
\end{tabular}

Fig. 2: Main parameters of HGTD [3].

inter-pad space, with a low capacitance of the detector, and this is relevant for the temporal resolution. For the read-out a custom low-noise ASIC ALTIROC [7], [8] is developed and bump-bonded to the sensors, to answer the demands for temporal resolution and radiation hardness. Another functionality of the ASIC will be to provide a non-biased bunch by bunch luminosity measurement, and the implementation of a trigger with minimal bias, by counting the amount of hits stored in the detector and deliver this data at $40 \mathrm{MHz}$. A 35 ps timeresolution at the start of HL-LHC (Run 4) is required, followed by a 70 ps time-resolution by the end of the HL-LHC run period. Fig. 2 summarize the main parameters of the HGTD.

\section{B. Layout}

The objective from the design of the detector is to ensure the optimal timing resolution to effectively remove the impact of the pile-up in the forward region. For this reason, each layer of the HGTD is double-sided, and each side is rotated in opposite directions by $15^{\circ}$. On a common cooling disk, modules with on-detector electronics and sensors are installed on both sides, front and back. The modules are arranged in an overlapping manner, with $20 \%$ overlap for the radius of the disk $r>470$ $\mathrm{mm}, 54 \%$ for $\mathrm{r} \in[230,470] \mathrm{mm}$ and $70 \%$ for $\mathrm{r}<230 \mathrm{~mm}$. The choice of the optimal overlap for each ring was based on a full simulation, in order to achieve the required timing resolution via the average number of simulated hits, given the expected time resolution of the pads.

\section{Radiation hardness}

The radiation hardness of the sensors and electronics is one of the most important parameters of the HGTD. To achieve sufficient performance of the sensors and ASICs, the detector layout has been designed considering a replacement scenario during the HL-LHC. Through an intensive R\&D campaign, a minimum charge of $4 \mathrm{fC}$ is required, this can be achieved up to a radiation damage of $2.5 \times 10^{15} \mathrm{n}_{e q} \mathrm{~cm}^{2}$ and total ionising dose (TID) about 2.0 MGy. As a result, the sensors and electronics within the lowest-radius $(\mathrm{r}<230 \mathrm{~mm})$ will be replaced after each $1000 \mathrm{fb}^{-1}$ and the sensors and ASICs within $230 \mathrm{~mm}<\mathrm{r}<470 \mathrm{~mm}$ should be replaced at half of the data-taking $\left(2000 \mathrm{fb}^{-1}\right)$ during the HL-LHC program. In the inner ring the total $\mathrm{Si} 1 \mathrm{MeV} \mathrm{n} \mathrm{n}_{e q}$ fluence has a similar proportion of charged particles and neutrons, whereas in the outer and central rings, the effect is dominated by neutrons. To account for uncertainties in the simulation, a total safety factor of 1.5 is applied for the sensors which are most sensitive to the fluence of the particles, and for the electronics which is more sensitive to TID, 2.25 as a factor. The maximum fluence and total ionising dose as a function of the radial position including the replacement of the rings can be found in Fig. 3.

\section{Module components}

The hybrid module is made of two parts: two LGAD sensors and two ASICs, called bare module, and the flexible printed circuit board (flex cables). The flex is made of two pieces, a small flex board permanently glued to the bare module and a long flex tail whose length, of up to about $60 \mathrm{~cm}$, depends on the module position in the detector. The sensors and the ASICs are connected through a flip-chip bump bonding process called hybridization. All connections between the ASIC and the peripheral electronics are routed through the flex cable. The bare module is glued on the back side of the sensor to the flex module small piece, and all the signals are wire bonded between the ASIC and the flex cable and for the high voltage between the sensor and the flex. The size of the bare modules is around $2 \times 4 \mathrm{~cm}^{2}$, the pads size is $1.3 \times 1.3 \mathrm{~mm}^{2}$. Each sensor is connected to one ASIC, reading a matrix of 225 channels. A view of the HGTD hybrid module is showed in the Fig. 4.

\section{SENSORS AND READOUT ELECTRONICS}

\section{A. LGAD sensors}

LGADs are n-on-p silicon detectors that contain an additional highly doped p-layer under the n-p junction to create a high field that causes internal amplifications referred as the gain of the LGAD as illustrated in Fig. 5. The gain is related to the dose of doping in the multiplier layer and reduces with the fluence of radiation. The HGTD LGADs were initially designed by the Centro Nacional de Microelectrnica (CNM) in Barcelona [6] and developed during the last 5 years within the CERN-RD50 community [9] including collaboration with two other LGAD vendors: Hamamatsu Photonics (HPK, Japan) and Fondazione Bruno Kessler (FBK, Italy).

Three major effects determine the time resolution: time walk from amplitude variations, jitter from electronic noise and 

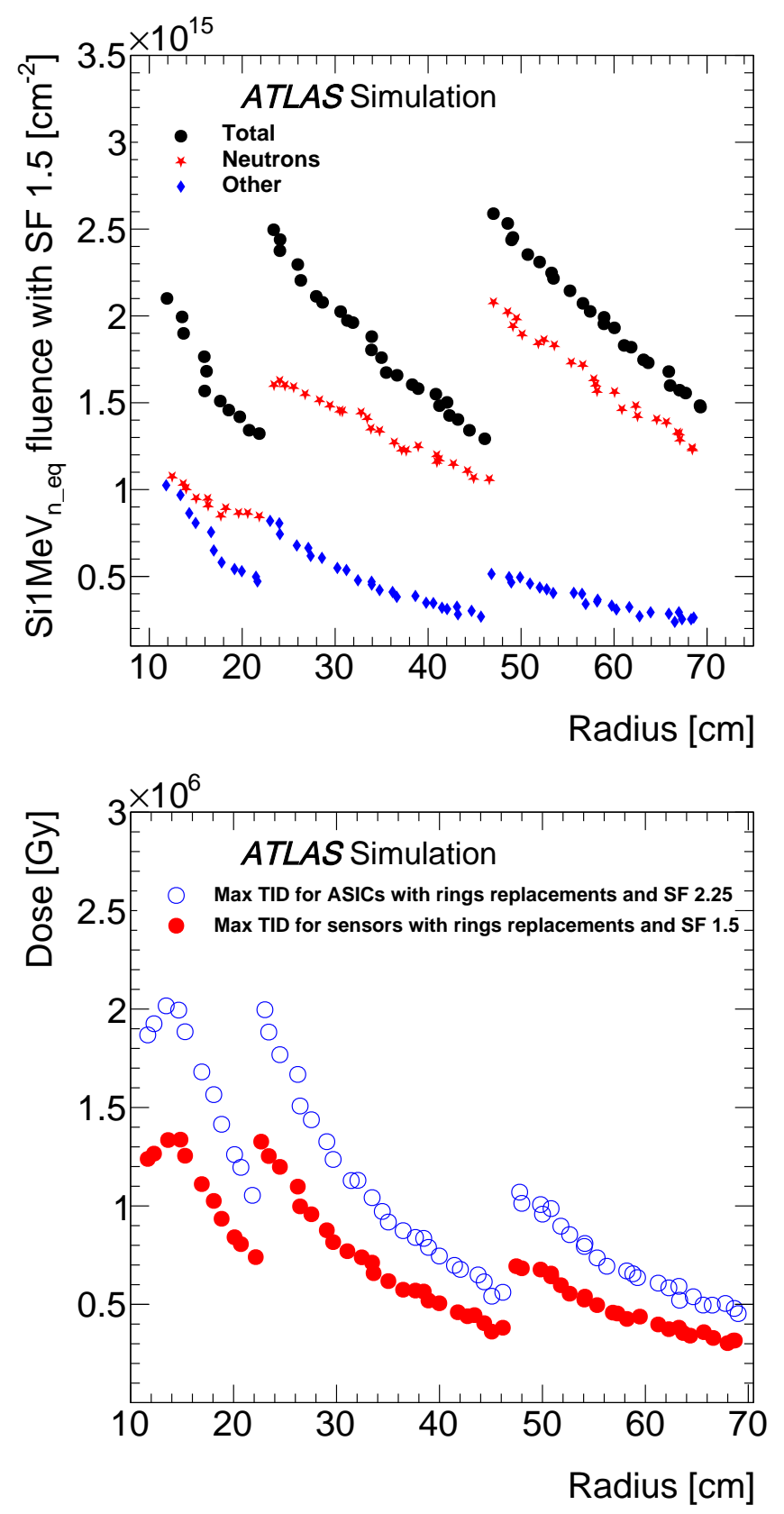

Fig. 3: Expected Si $1 \mathrm{MeV} \mathrm{n}_{e q}$ radiation levels in HGTD, using Fluka simulations, as a function of the radius considering a replacement of the inner ring every $1000 \mathrm{fb}^{-1}$ and the middle ring replaced at $2000 \mathrm{fb}^{-1}$ [3].

Landau fluctuations from charge deposition non-uniformities along the particle path. Time walk and noise jitter depend on the type of readout electronics chosen. Both depend inversely on the signal slope (voltage slope at the output of the amplifier) $\mathrm{dV} / \mathrm{dt}$ :

$$
\begin{gathered}
\sigma_{\text {TimeWalk }}=\left[\frac{V_{t h}}{\frac{S}{t_{\text {rise }}}}\right]_{R M S} \propto\left[\frac{N}{\frac{d V}{d t}}\right]_{R M S^{\prime}} \\
\sigma_{\text {Jitter }}=\frac{N}{\frac{d V}{d t}} \simeq \frac{t_{\text {rise }}}{\frac{S}{N}}
\end{gathered}
$$

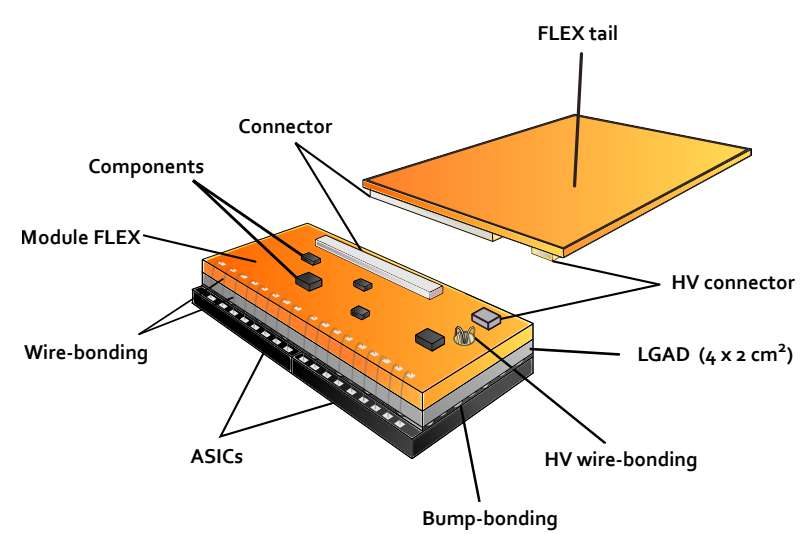

*not to scale

Fig. 4: View of an HGTD hybrid module equipped with its read-out flex cable tail [3].

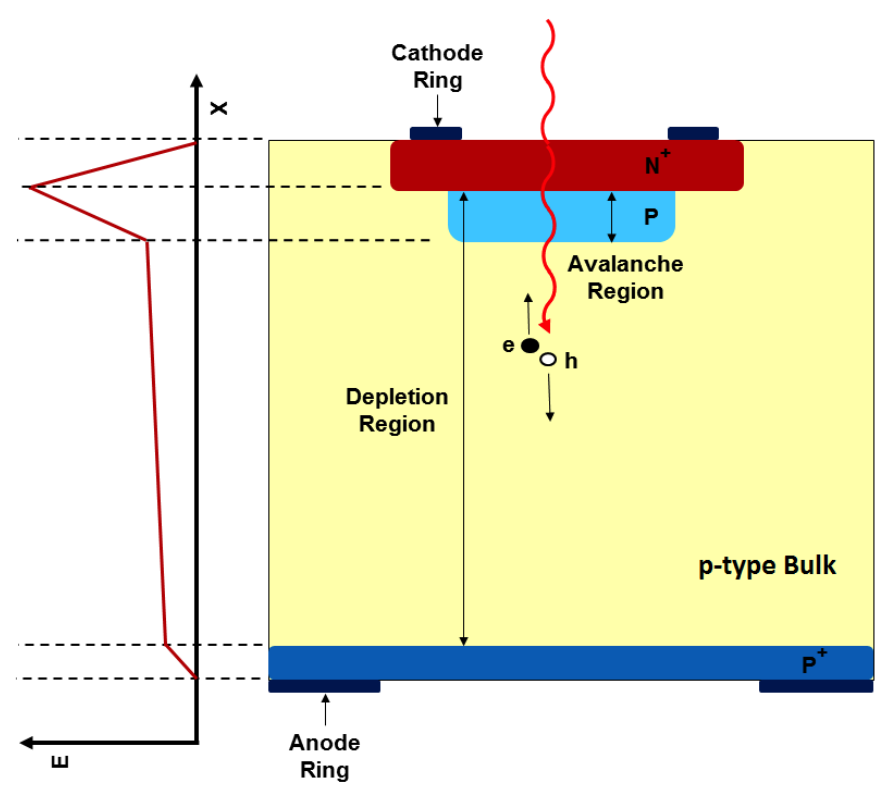

Fig. 5: View of the cross section of an LGAD [3].

where $\mathrm{S}$ refers to the signal which is proportional to the gain, $\mathrm{V}_{t h}$ to the threshold voltage, $\mathrm{t}_{\text {rise }}$ to the rise time and $\mathrm{N}$ to the noise. The third effect, referred to Landau fluctuation is due to the non-uniform charge deposition along the particle path leading to time-of-arrival fluctuations. It is a contribution depending on the thickness of the sensor (thin is beneficial) and the setting of the threshold. Adding the three contributions in quadrature yields the overall time resolution. After time walk correction, the noise jitter is the dominating contribution for low S/N and the Landau term takes over for high $\mathrm{S} / \mathrm{N}$.

\section{B. Electronics readout}

Each LGAD will be read out using the ASIC chip, ALTIROC that will have 225 readout channels. Two iterations of this chip have been produced and tested so far: the first, ALTIROC0, integrated four pads in a $2 \times 2$ array, with the ana$\log$ part of the single-channel readout: the preamplifier and the 


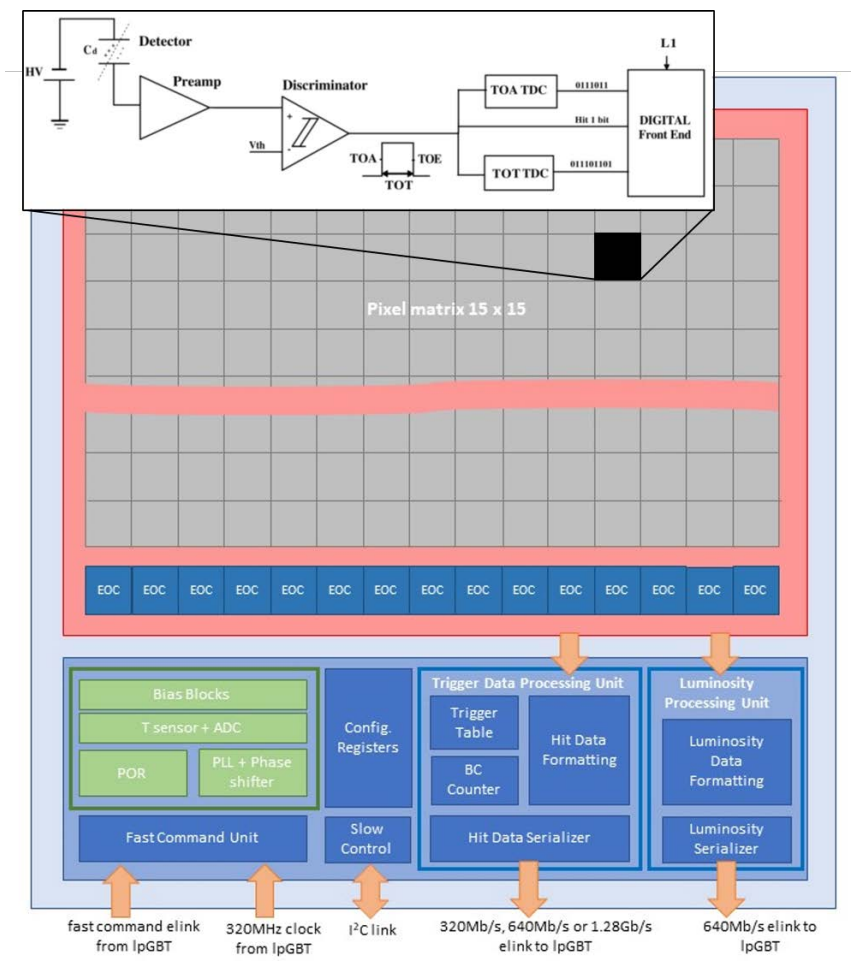

Fig. 6: Global architecture of the ALTIROC ASIC. The schematic of one Front End electronics channel is displayed on top of the channels matrix, with the preamplifier followed by a discriminator, two TDCs, and a digital front end block. The ASIC common digital part is shown in the bottom [3].

discriminator. The second iteration, ALTIROC1, consists of a $5 \times 5$ pad matrix, in which the digital components have been added to the single-channel readout. The next major ASIC iterations, ALTIROC2, will integrate all the functionality of the final ASIC and will have its final size, and the ALTIROC3 which will be the radiation hard version of the ALTIROC2. The ASIC chip will provide the Time Of Arrival (TOA), with measurements restricted to a $2.5 \mathrm{~ns}$ window centered on the bunch crossing, and the Time Over Threshold (TOT) used offline to correct the TOA for time walk effect in order to achieve the target time resolution with an ASIC contribution smaller than $25 \mathrm{ps}$, and its discriminator threshold expected to be about $2 \mathrm{fC}$. The design of the whole ASIC of the HGTD with 225 channels is shown in the Fig. 6.

\section{SENSORS MEASUREMENTS AT LABORATORY}

The dynamic characteristics of LGADs, like the temporal resolutions, gain and charge collection have been determined in the laboratory with $\mathrm{Sr}^{90} \beta$ particles in response to ionizing particles. Fig. 7 illustrates the charge collected as a function of the biasing voltage for different fluences for HPK-3.2 (top) and at maximum fluence for all vendors (bottom). The horizontal lines indicate the HGTD lower charge limit of $4 \mathrm{fC}$ at all fluences. Solid markers indicate neutron irradiation (n), open markers $\mathrm{p}$ irradiation at CYRIC (pCy). Measurements were performed at $-30{ }^{\circ} \mathrm{C}$ except for the pre-rad measurement that was done at $20^{\circ} \mathrm{C}$. The HGTD requirement to collect up to 4
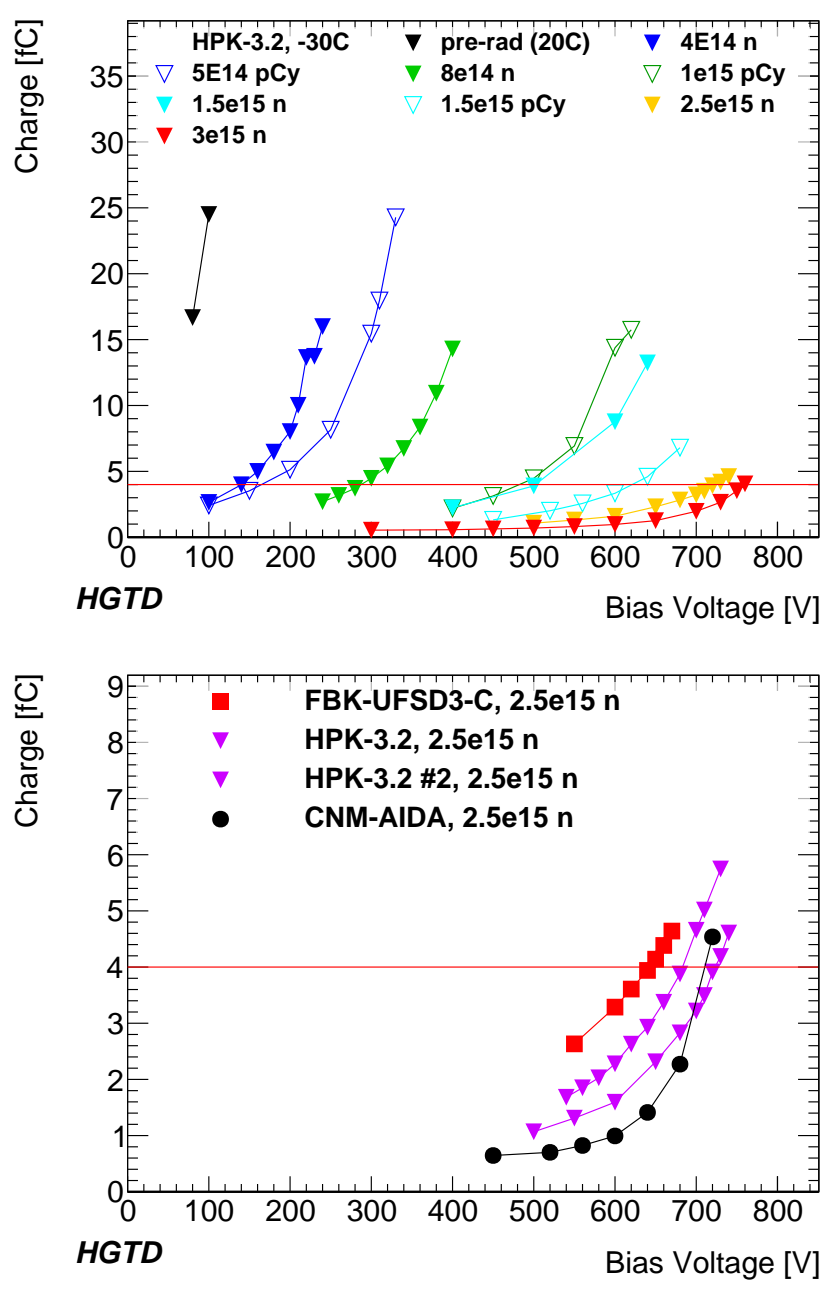

Fig. 7: Collected charge as a function of bias voltage for different fluences for HPK-3.2 (top) and at maximum fluence for all vendors (bottom) [10].

fC for efficient HGTD operation until the end of the HL-LHC phase has been met by these sensors.

\section{TEST BEAM CAMPAignS}

To study the LGAD performance before and after irradiation, and the HGTD modules, the sensors have been exposed to charged-particle beams. The HGTD community performed the test beam in more than fifteen periods between 2016 and 2020 at the H6 beam line of the CERN SPS [11] with 40 to 120 $\mathrm{GeV}$ pions, at Fermilab with $120 \mathrm{GeV}$ protons, at SLAC with $15 \mathrm{GeV}$ electrons, and at DESY with $5 \mathrm{GeV}$ electrons [12], [13]. In the next sections, we will present the experimental setup using by the DESY laboratory as a model, and the test beam results for different laboratories.

\section{A. Experimental setup}

An EUDET-type [14] telescope based on MIMOSA pixel planes was used to provide the position-dependent measurements. Also for the triggering purposes, an additional pixel 


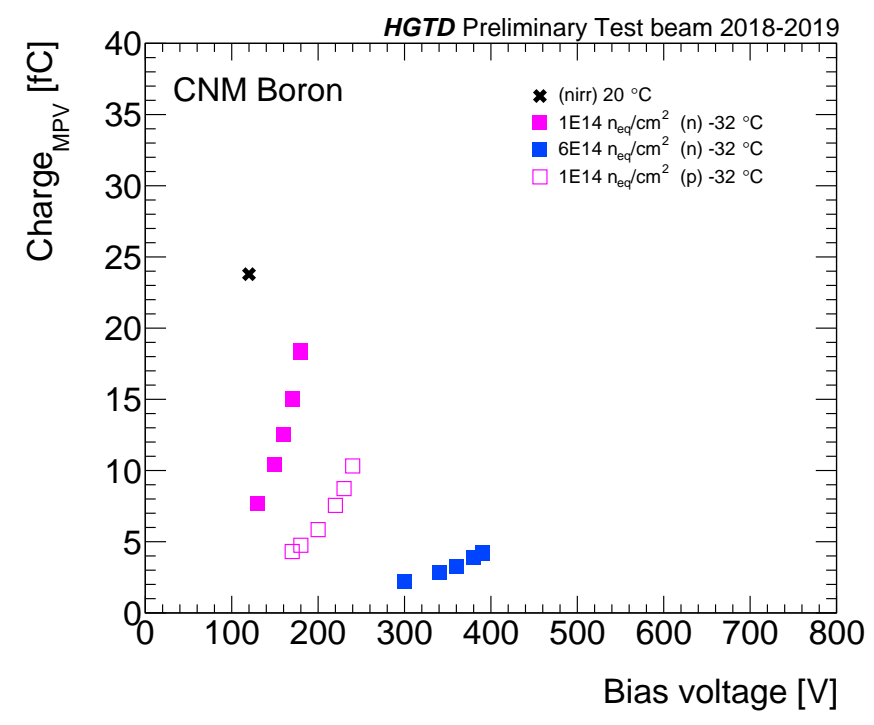

Fig. 8: Collected charge as a function of the the bias voltage for different single pad sensors built by CNM [10].

reference plane was employed, using the FE-I4 [15] readout chip. The signals of the trigger were combined in the Trigger Logic Unit (TLU), whose output was used by the telescope and connected to the oscilloscope, ensuring a perfect match between the events recorded by the telescope and the oscilloscope(s). In addition Cherenkov light emitting quartz bars coupled to Silicon Photo-multipliers (SiPM) were used as timing references. The installation was assembled on a plate base linked to a remote controlled stage motors that moved in both vertical and horizontal axes orthogonal to the direction of the beam with a micrometer accuracy. The polystyrene container that covers the devices under test has been used to ensure both the light-tightness and to stabilize the temperature to about $25^{\circ} \mathrm{C}$ with a water cooled system. To prevent condensation of moisture that may harm the sensors, a locally available nitrogen outlet was added to the box.

\section{B. Results}

Fig. 8 shows the collected charge as a function of the the bias voltage for different single pad sensors built by CNM from wafer W4 produced in the run 10478 , doped with Boron. Measurements were performed at $-32{ }^{\circ} \mathrm{C}$ for sensors irradiated with neutrons (at $1 \times 10^{14} \mathrm{n}_{e q} / \mathrm{cm}^{2}$ and $6 \times 10^{14} \mathrm{n}_{e q} / \mathrm{cm}^{2}$ ) or with protons (empty markers) at $1 \times 10^{14} \mathrm{n}_{e q} / \mathrm{cm}^{2}$ and at 20 ${ }^{\circ} \mathrm{C}$ for the unirradiated one. The fluences are provided with a precision of $10 \%$. At every event, the collected waveform charge is computed as the area integral of the signal. Using the Landau-Gauss convoluted function, the distribution of the charge is fitted, where the charge collected is determined to be the likeliest value of the fitted function. After irradiating this sensor to $6 \times 10^{14} \mathrm{n}_{e q} / \mathrm{cm}^{2}$, it achieves the $4 \mathrm{fC}$ value that is demanded for an ALTIROC optimal performance, and specifically $4.2 \mathrm{fC}$ at $390 \mathrm{~V}$.

The Constant Fraction Discriminator (CFD) have been chosen in order to optimise time resolution. Using this algorithm, for every single event in a run, the waveform TOA is calculated

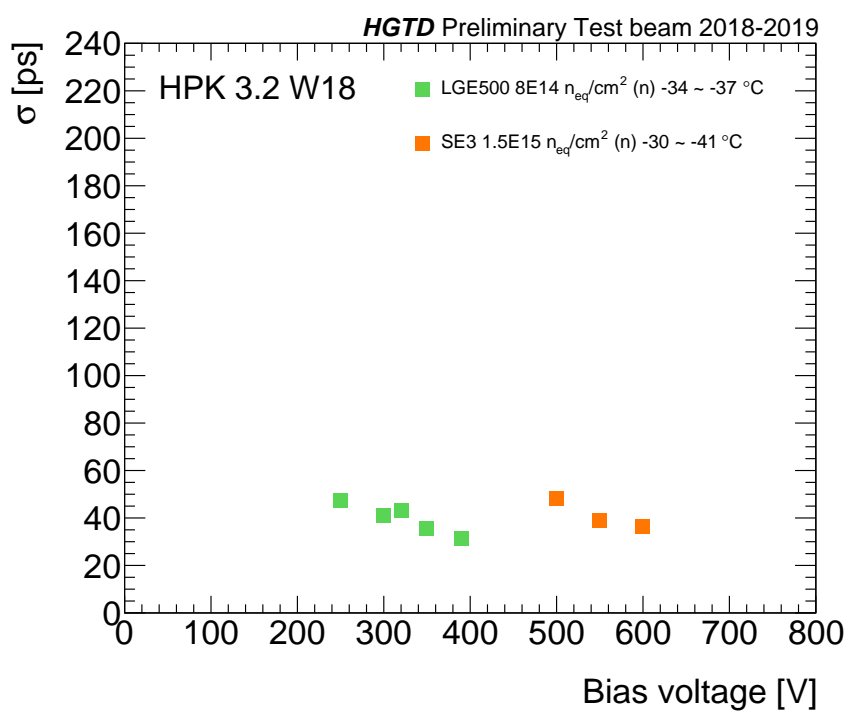

Fig. 9: Time resolution as a function of the bias voltage for different single pad sensors built by HPK [10].

as the time of crossing a given threshold. To determine this threshold we use the CFD fraction from the signal amplitude, defined using the time resolution dominant factor. For unirradiated sensors, the Landau effect is the dominant, whereas is the noise in irradiated sensor. In order to provide time reference signal we used a non-irradiated LGAD. The time resolution was calculated by fitting the distribution of the difference between time at a CFD $=50 \%$ for the device under test (DUT) and the time at a CFD $=20 \%$ for non-irradiated sensor. As it is shown in Fig. 9 the HPK-3.2 sensors doped with Boron irradiated to $1.5 \times 10^{15} \mathrm{n}_{e q} / \mathrm{cm}^{2}$ reaches the HGTD timing requirement with $36 \mathrm{ps}$, at $600 \mathrm{~V}$ for a collected charge of $22.8 \mathrm{fC}$.

The hit reconstruction efficiency is determined by calculating the ratio between the number of tracks that trigger the sensor response (with a collected charge greater than $2 \mathrm{fC}$ ) and the total number of reconstructed tracks traversing the DUT at a given position. Fig. 10 (top) manifest the efficiency as a function of bias voltage for different single pad sensors built by CNM from wafer W6 produced in the run 10924, doped with Gallium. Sensors were irradiated with neutrons or protons with different fluences. The efficiency is computed for a charge threshold of $2 \mathrm{fC}$ which corresponds to the threshold of the ALTIROC and from hits in the pad center region (in $0.5 \times 0.5$ $\mathrm{mm}^{2}$ area). For CNM sensors doped with gallium irradiated to $3 \times 10^{15} \mathrm{n}_{e q} / \mathrm{cm}^{2}$ the efficiency is about $99.7 \%$ for the bias voltage of $740 \mathrm{~V}$ and with $5.3 \mathrm{fC}$ as collected charge. The bottom plot of Fig. 10 shows the efficiency 2D map for the same sensor with same voltage. This map confirms that even after irradiation the sensor still very efficient in the center, while the borders are less efficient.

\section{CONCLUSION}

The addition of the timing information is a powerful new tool to improve the performance of the HL-LHC detectors. Therefore, the HGTD detector has been proposed to meet the 

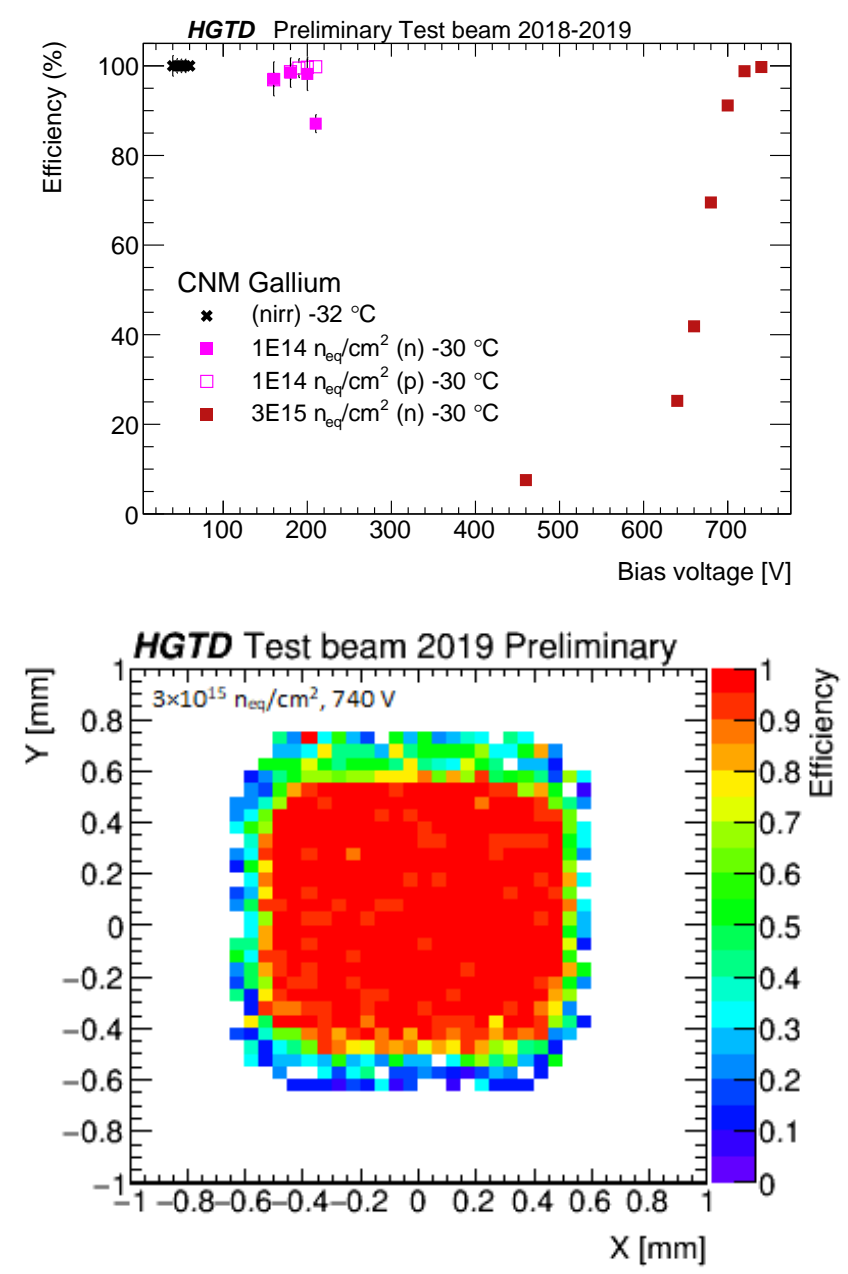

Fig. 10: Efficiency as a function of bias voltage for different single pad sensors built by CNM (top), Signal efficiency in a 1 x $1 \mathrm{~mm} 2$ CNM Gallium-doped LGAD irradiated to $3 \mathrm{e} 15$ $\mathrm{neq} / \mathrm{cm} 2$, as a function of the reconstructed position $\mathrm{X}$ and $\mathrm{Y}$ of particles (in mm) (bottom) [10].

requirements of the forward region. This detector is planned to be built using LGAD sensor technology, which will provide a time resolution of $30-50$ ps per track until the end of the HL-LHC lifetime. The R\&D carried out shows that HGTD will improve the physical performance of the ATLAS detector in the dense environment of the HL-LHC. In addition, the technology development opens up new possibilities for the application of LGAD detectors in fields other than particle physics.

\section{REFERENCES}

[1] ATLAS Collaboration, High-Luminosity Large Hadron Collider (HLLHC): Technical Design Report V. 0.1, ser. CERN Yellow Reports: Monographs. Geneva: CERN, 2017. [Online]. Available: http: //cds.cern.ch/record/2284929

[2] ATLAS Collaboration, "Technical Design Report for the ATLAS Inner Tracker Pixel Detector," CERN, Geneva, Tech. Rep., Sep 2017. [Online]. Available: https://cds.cern.ch/record/2285585

[3] ATLAS Collaboration, "Technical Design Report: A High-Granularity Timing Detector for the ATLAS Phase-II Upgrade," CERN, Geneva, Tech. Rep., Jun 2020. [Online]. Available: https://cds.cern.ch/record/ 2719855
[4] ATLAS Collaboration, "The ATLAS Experiment at the CERN Large Hadron Collider," JINST, vol. 3, p. S08003. 437 p, 2008, also published by CERN Geneva in 2010. [Online]. Available: https://cds.cern.ch/record/1129811

[5] ATLAS Collaboration, "ATLAS Liquid Argon Calorimeter Phase-I Upgrade: Technical Design Report," Tech. Rep., Sep 2013, final version presented to December 2013 LHCC. [Online]. Available: https://cds.cern.ch/record/1602230

[6] G. Pellegrini, P. Fernández-Martínez, M. Baselga, C. Fleta, D. Flores, V. Greco, S. Hidalgo, I. Mandić, G. Kramberger, D. Quirion, and M. Ullan, "Technology developments and first measurements of Low Gain Avalanche Detectors (LGAD) for high energy physics applications," Nuclear Instruments and Methods in Physics Research Section A: Accelerators, Spectrometers, Detectors and Associated Equipment, vol. 765, pp. 12-16, 2014. [Online]. Available: https://www.sciencedirect.com/science/article/pii/S0168900214007128

[7] C. Agapopoulou, S. Blin, A. Blot, L. Castillo García, M. Chmeissani, S. C. Di Lorenzo, C. de La Taille, P. Dinaucourt, A. Fallou, J. G. Rodriguez et al., "Performance of a front end prototype asic for picosecond precision time measurements with lgad sensors," Journal of Instrumentation, vol. 15, no. 07, p. P07007, 2020.

[8] C. de la Taille, S. Callier, S. Conforti, P. Dinaucourt, G. MartinChassard, N. Seguin-Moreau, C. Agapopoulou, N. Makovec, L. Serin, and S. Simion, "ALTIROC0, a 20 pico-second time resolution ASIC for the ATLAS High Granularity Timing Detector (HGTD)," $P o S$, vol. TWEPP-17, p. 006, Sep 2017. [Online]. Available: https://cds.cern.ch/record/2319292

[9] RD50 Collaboration. [Online]. Available: https://rd50.web.cern.ch/rd50

[10] ATLAS HGTD Public Plots. [Online]. Available: https://twiki.cern.ch/ twiki/bin/view/AtlasPublic/HGTDPublicPlots

[11] C. Allaire, J. Benitez, M. Bomben, G. Calderini, M. Carulla, E. Cavallaro, A. Falou, D. Flores, P. Freeman, Z. Galloway, E. Gkougkousis, H. Grabas, S. Grinstein, B. Gruey, S. Guindon, A. Henriques Correia, S. Hidalgo, A. Kastanas, C. Labitan, D. Lacour, J. Lange, F. Lanni, B. Lenzi, Z. Luce, N. Makovec, G. Marchiori, L. Masetti, A. Merlos, F. McKinney-Martinez, I. Nikolic-Audit, G. Pellegrini, R. Polifka, D. Quirion, A. Rummler, H. Sadrozinski, A. Seiden, L. Serin, S. Simion, E. Spencer, S. Trincaz-Duvoid, M. Wilder, A. Zatserklyaniy, D. Zerwas, and Y. Zhao, "Beam test measurements of Low Gain Avalanche Detector single pads and arrays for the ATLAS High Granularity Timing Detector," JINST, vol. 13, p. P06017. 28 p, Apr 2018. [Online]. Available: https://cds.cern.ch/record/2313951

[12] R. Diener, J. Dreyling-Eschweiler, H. Ehrlichmann, I. M. Gregor, U. Kötz, U. Krämer, N. Meyners, N. Potylitsina-Kube, A. Schütz, P. Schütze, and M. Stanitzki, "The DESY II test beam facility," Nuclear Instruments and Methods in Physics Research Section A: Accelerators, Spectrometers, Detectors and Associated Equipment, vol. 922, pp. 265-286, 2019. [Online]. Available: https://www.sciencedirect. $\mathrm{com} / \mathrm{science} / \mathrm{article} / \mathrm{pii} / \mathrm{S} 0168900218317868$

[13] L. Castillo Garcia, "A High-Granularity Timing Detector for the Phase-II upgrade of the ATLAS Calorimeter system: detector concept, description and R\&D and beam test results," CERN, Geneva, Tech. Rep., May 2020. [Online]. Available: https://cds.cern.ch/record/2717972

[14] H. Jansen, S. Spannagel, J. Behr, A. Bulgheroni, G. Claus, E. Corrin, D. Cussans, J. Dreyling-Eschweiler, D. Eckstein, T. Eichhorn et al., "Performance of the eudet-type beam telescopes," EPJ Techniques and Instrumentation, vol. 3, pp. 1-20, 2016. [Online]. Available: https: //link.springer.com/content/pdf/10.1140/epjti/s40485-016-0033-2.pdf

[15] U. Koetz and I. Rubinskiy, "User manual: Atlas fe-i4a pixel module as a trigger plane for the beam telescope," 2013. [Online]. Available: https://telescopes.desy.de/images/0/08/131014_ koetz-rubinsky_FEI4_as_trigger_plane.pdf 